\title{
MONITORIA ACADÊMICA EM OPERAÇÕES DE SEPARAÇÃO I NO PERÍODO DE ENSINO REMOTO: RELATO DE EXPERIÊNCIA
}

DOI: 10.37702/2175-957X.COBENGE.2021.3563

Ana Beatriz da Silva dos Santos - beatrizsilva.qi@gmail.com

Universidade Federal do Maranhão

Rua Cristino de Oliveira 27

65058-844 - São Luís - MA

Kátia Simone Teixeira da Silva de La Salles - katia.salles@ufma.br Universidade Federal do Maranhão

AV. sao Luis rei de franca 48

65065-470 - sao luis - MA

Resumo: A pandemia provocada pelo novo coronavírus (Sars-Cov-2) provocou sérias mudanças em todos os setores da sociedade. Dentre elas, no ambiente acadêmico, houve a suspensão das atividades letivas presenciais e a consequente migração para o ensino remoto emergencial. $O$ presente artigo tem por objetivo descrever, através de um relato de experiência, os benefícios e desafios encontrados na monitoria no ensino remoto, e uma breve avaliação da percepção dos estudantes monitorados na disciplina Operações de Separação I, ministrada aos discentes dos cursos graduação em Química Industrial e Engenharia Química de uma Instituição de Ensino Superior do Estado do Maranhão, entre fevereiro e abril de 2021. Nesse período, devido à pandemia, as atividades foram desenvolvidas através de Tecnologias da Informação e Comunicação (TIC), com apoio de ferramentas do Google Meet, Google Classroom e Whatsapp, que possibilitaram minimizar as lacunas em virtude da impossibilidade dos encontros presenciais. Como monitora em um cenário inesperado, foi possível vivenciar novos aprendizados, aprofundar e obter novos conhecimentos gerando crescimento pessoal e profissional, construção das relações interpessoais, e desenvolvimento da empatia.

Palavras-chave: Monitoria. Ensino. Processos. Operações de Separação. 


\section{MONITORIA ACADÊMICA EM OPERAÇÕES DE SEPARAÇÃO I NO PERÍODO DE ENSINO REMOTO: RELATO DE EXPERIÊNCIA}

\section{INTRODUÇÃO}

A pandemia provocada pelo novo coronavírus (Sars-Cov-2), já vitimou centenas de milhares de pessoas no planeta. Além de causar um colapso no sistema de saúde de vários países, provocou também mudanças radicais em todos os demais setores da sociedade impondo uma nova realidade nas atividades do dia a dia, bem como no ambiente acadêmico. Como efeito, houve a suspensão das atividades letivas presenciais por todo o mundo, o que obrigou docentes e discentes a migrarem para a realidade online, transferindo e transpondo metodologias e práticas pedagógicas típicas das salas de aulas físicas de aprendizagem para o ensino remoto emergencial (MOREIRA; HENRIQUES; BARROS, 2020).

Com a adoção desta nova modalidade de ensino, surgiram vários desafios a serem superados. Problemas com conexão à internet, a busca por aplicativos e sites inovadores para a criação de conteúdos e execução de provas e trabalhos de forma diferenciada e mais atrativa, realização da frequência individual nas atividades, e entre diversas outras situações foram adicionadas à lista de responsabilidades dos docentes (MARQUES, et al. 2021). E, assim como os professores tiveram que se remodelar ao ambiente virtual, o mesmo ocorreu para os discentes que oferecem monitoria dos componentes curriculares.

A atividade de monitoria, solidificada no Brasil por meio da lei federal $n^{\circ} 5.540$, de 28 de novembro de 1968, pode ser definida como um processo pelo qual estudantesmonitores, de acordo com seu rendimento e seu plano de estudo, auxiliam seus colegas de curso em processos de ensino-aprendizagem, sob orientação direta de um professor. A monitoria é um instrumento facilitador de aprendizagem, sendo realizado por estudante e para os estudantes com o objetivo de transferir conhecimento e auxiliar o professor em suas atividades.

Com efeito, a monitoria é um suporte importante aos estudantes, por esclarecer questionamentos e possibilitar maior êxito na disciplina em estudo.

O contato direto com os estudantes, presencialmente, na condição de iguais, possibilitava ao monitor experienciar situações únicas inerentes à docência, que vão desde a alegria de contribuir, pedagogicamente, com o aprendizado de alguns, até a momentânea desilusão em situações em que a conduta de alguns alunos se mostrava inconveniente e desestimuladora (ASSIS, et al. 2006).

No entanto, as atividades de monitoria tiveram que ser adaptadas ao uso de Tecnologias de Informação e Comunicação (TIC) na tentativa de continuar o programa, e de forma a minimizar as lacunas no aprendizado dos alunos no cenário atual (CORONEL; GUEDES; PIRANDA, 2020).

Dentro dessa perspectiva, o presente artigo tem por objetivo apresentar a importância, os benefícios, e os desafios e soluções encontrados na monitoria no ensino remoto, e uma breve avaliação da percepção dos estudantes monitorados na disciplina Operações de Separação I ministrada aos discentes dos cursos de bacharelado em Química Industrial e Engenharia Química na Universidade Federal do Maranhão (UFMA). 


\section{METODOLOGIA}

Trata-se de um estudo descritivo, do tipo relato de experiência, realizado a partir da vivência discente na monitoria da disciplina Operações de Separação I, que, no curso de bacharelado em Química Industrial da UFMA, é ofertada a discentes do $7^{\circ}$ período, conforme projeto pedagógico do curso. Tal experiência ocorreu no município de São Luís/MA, no período de fevereiro a abril de 2021, correspondendo ao período 2020.2.

O presente trabalho utilizou-se de uma pesquisa de campo quantitativa-descritiva através de formulário preenchido pelos alunos monitorados. O formulário foi desenvolvido na plataforma Google Form e seu preenchimento foi anônimo e voluntário. Também foram usados artigos a respeito da monitoria no âmbito acadêmico e sobre os desafios da educação no ensino remoto.

A disciplina de Operações de Separação I propõe uma análise qualitativa de algumas operações de separação da indústria química, dando ênfase aos fundamentos que norteiam estas operações, apresentando os princípios de funcionamento dos equipamentos industriais e o impacto operacional das principais variáveis operacionais inerentes ao processo em estudo. Entre as operações abordadas pela disciplina, encontram-se as operações envolvendo sistemas sólidos granulares, sistemas fluidos, sistemas sólidofluido, e separações mecânicas.

Coube à monitoria auxiliar os discentes com esclarecimentos de eventuais dúvidas sobre os diversos conteúdos ofertados na disciplina, de modo a facilitar o processo ensinoaprendizagem e orientá-los na realização das atividades propostas por meio de encontros virtuais em atendimento extraclasse via WhatsApp e Google Meet; desenvolver e implantar, em conjunto com o professor responsável pela disciplina, práticas de metodologias ativas nas componentes curriculares trabalhadas (elaboração de desafios e jogos); incentivar a interação professor-aluno e participação mais ativa dos discentes nas aulas virtuais.

\section{RESULTADOS E DISCUSSÃO}

Dentre todas as experiências oferecidas ao longo de uma graduação, a monitoria acadêmica é, certamente, uma das mais enriquecedoras. Seu valor excede ao caráter de obtenção de um título ou uma simples complementação acadêmica. O privilégio oferecido aos aprovados nos programas de monitoria é de fundamental importância para a descoberta da vocação, ou não, pela docência, pois possibilita ao discente vivenciar uma possível escolha de carreira, evitando, assim, que, no futuro, possam tornar-se profissionais descontentes (MATOSO, 2014).

A monitoria oportuniza ao estudante viver em duas realidades distintas simultaneamente. Permite que o mesmo transite entre o discente e o docente, atuando como um intermediador e um incentivador. $O$ estudante, quando inserido neste processo, desempenha funções próximas à docência, de forma amadora, através de assistência dada aos alunos monitorados, sendo um facilitador no processo ensino-aprendizagem.

A presente monitoria deu-se inicialmente por um processo de seleção entre os alunos interessados do curso de Química Industrial da UFMA no qual, para minha surpresa, não houve outras candidaturas. Após breve reflexão, foi possível perceber que a pouca procura foi devido à dificuldade de adequação ao ensino remoto tendo que lidar com todas as disciplinas decorrentes no semestre e conciliá-las junto a monitoria, e à dificuldade com a disciplina em si. Isto porque trata-se uma disciplina extensa e complexa que, no período 
remoto, estuda-se de forma breve, não sendo possível aprofundar o conhecimento mesmo com todo o esforço da docente em transmitir o conteúdo, utilizando estratégias ativas como mapas mentais, podcasts e vídeo para que os alunos pudessem se desenvolver e fixar melhor o assunto. Diante disto, o desafio proposto ao assumir a monitoria tornava-se ainda mais interessante, visto que havia muito apreço da monitora pela disciplina e pela área dos processos industriais.

Findados os procedimentos para o início do programa e os concordes com a docente no que tange às atribuições da monitora, a mesma foi apresentada pela docente aos alunos via sala do Google Classroom, e foram acertados os horários e plataformas em que os estudantes monitorados poderiam solicitar ajuda.

Durante o exercício da monitoria, o acompanhamento realizado pelo professor orientador é essencial, pois, todo o suporte e orientação concedidos ao monitor lhe conferem mais segurança, autonomia e tranquilidade no exercício da função, deixando de lado a insegurança, a ansiedade e o medo da frustração. No decurso deste programa, todo o apoio e compreensão doados pela docente tornaram-se fundamentais para a realização das atividades de monitoria e formação da monitora.

Embora tenha sido em apenas 3 meses, o programa proporcionou diversos benefícios como a revisão e aprimoramento dos conteúdos abordados na disciplina estudada anteriormente, a ampliação da relação interpessoal com a docente e os alunos monitorados, maior compreensão das adversidades e preocupações que sobrevém aos docentes em relação aos alunos, a possibilidade de desenvolver produções científicas, e o enriquecimento do currículo acadêmico.

Contudo, a mudança do presencial para o remoto também trouxe consigo diversas dificuldades. Na tutoria EaD, a autoavaliação enquanto transmissora de conhecimento foi uma das mais marcantes, pois em todo o processo didático foi necessário o policiamento das palavras escolhidas para dar o máximo de clareza e coerência às explicações, observando como o aluno estava recebendo a informação, e se o mesmo a estava entendendo, o que seria mais simples de observar se estivéssemos no mesmo ambiente, em uma sala de aula. Além disso, todos tiveram que lidar com diferentes problemas como a dificuldade em manter a atenção nas atividades, o acesso à internet, acesso à aparelhos tecnológicos como computadores, tablets, celulares para o acompanhamento das aulas e, até mesmo a própria situação de pandemia que provocou uma mudança repentina na rotina de vida de todos (SILVA; DA CRUZ, 2021).

Sob essa perspectiva, o monitor precisa ser paciente, dinâmico, ativo e muito flexível. Uma das situações que intercorreram foi a tentativa de incentivar e motivar alguns estudantes que já estavam cansados mentalmente e indispostos não apenas para a disciplina. Os estudantes que outrora compartilhavam das alegrias e tristezas acadêmicas presencialmente, em um instante se depararam com a dura realidade do confinamento, e tiveram que adaptar-se à nova rotina de estudos, e de cuidados com a saúde mental e física. Como por exemplo, ocorreu de um dos estudantes que estava realizando uma das avaliações ficar sem internet, pois este usava dados móveis. Ele relatou que assim que sua internet acabou, dirigiu-se imediatamente à farmácia para recolocar os créditos que não caíram em tempo hábil de enviar a prova no tempo pré-estabelecido. Com isto, o aluno sentiu-se totalmente desmotivado a enviar a avaliação alguns minutos depois do prazo. Após tomar ciência disto, a monitora junto a professora, solicitaram ao aluno o envio da 
avaliação, visto que o ocorrido foi uma situação incomum, comprovada, e o estudante estava à beira da desistência.

Outra dentre as diversas dificuldades que permearam a atuação da monitoria, foi a falta de interesse de alguns discentes em procurar o auxílio. A atuação do monitor contribui significativamente para o aprendizado dos estudantes devido à possibilidade de maior interação e a facilidade da comunicação com o monitor, pois este também é estudante. No entanto, a prática exigiu uma postura mais séria para saber lidar, muitas vezes, com alunos que estavam angustiados, decorrente da necessidade de aprenderem, bem como para elaborar ferramentas pedagógicas capazes de estimular a busca dos discentes pelo monitor.

O número de estudantes que responderam à pesquisa foi num total 16 , de uma turma composta por 19 estudantes. Os resultados da avaliação são apresentados neste trabalho em forma de gráficos para uma melhor interpretação e visualização dos resultados das respostas coletadas no questionário.

A Figura 1 apresenta o resultado da avaliação de satisfação dos estudantes quanto a contribuição da monitoria no seu aprendizado.

Figura 1: Respostas dos alunos à pergunta: Numa escala de 1 a 10, sendo 1- nada importante e 10 - muito importante, em quanto você avalia a contribuição da monitoria ao seu aprendizado?

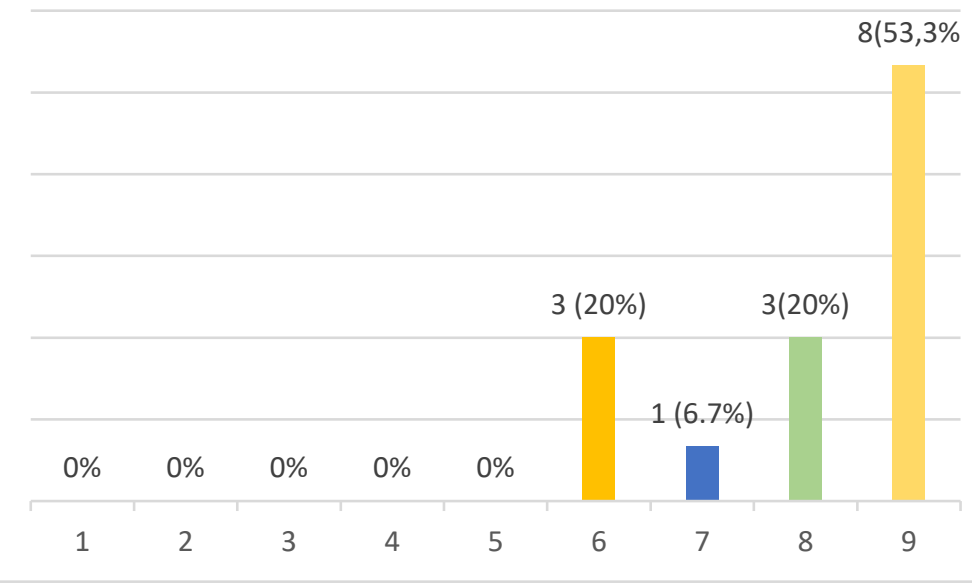

Fonte: Autora, 2021

Os dados obtidos mostraram que os estudantes, em sua maioria, acreditam que a monitoria contribuiu para o seu aprendizado (FIGURA 1), sendo que $73,3 \%$ dos estudantes atribuíram notas 9 e 10 para a contribuição da monitoria no seu aprendizado, em uma escala de 0 a 10, onde 0 significa nada importante e 10, muito importante. Este resultado é um indicador de quão promissor foi a contribuição da monitoria no aprendizado dos estudantes durante o período pandêmico.

Neste mesmo formulário buscou-se entender quais os fatores que mais influenciam à decisão de procurar, ou não, a ajuda da monitora. As respostas obtidas encontram-se na Figura 2.

Figura 2: Respostas dos alunos à pergunta: quais os fatores que mais influenciam à decisão de procurar ou não procurar a ajuda da monitora? 
Tempo de resposta da monitora.

Didática da monitora.

Conhecimento de quem é a monitora.

Interesse do aluno pela disciplina.

Desempenho do aluno na disciplina.

Preferência do aluno por estudar sozinho.

Conhecimento do monitor sobre a disciplina.

Disponibilidade da monitora.

Disponibilidade de horários do aluno.

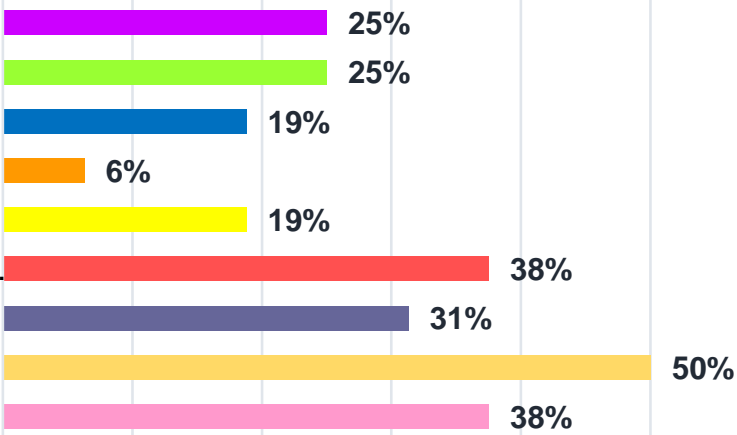

Fonte: Autora, 2021.

Podemos observar à partir da Figura 2 que dentre os fatores elencados pelos estudantes, os mais votados são relacionados ao tempo e ao conhecimento sobre a disciplina, isto para os que optaram pelo auxílio junto a monitora. Para os que não utilizaram-se da monitoria, o gráfico aponta que o principal motivo foi a preferência por estudar sozinho ou com os colegas, sendo os demais fatores considerados secundários.

Um outro aspecto investigado foi o desempenho da monitora, cujos resultados estão apresentados na Figura 3.

Figura 3: Desempenho da monitora

Qual o seu grau de satisfação em relação ao desempenho do monitor na disciplina? 16 respostas
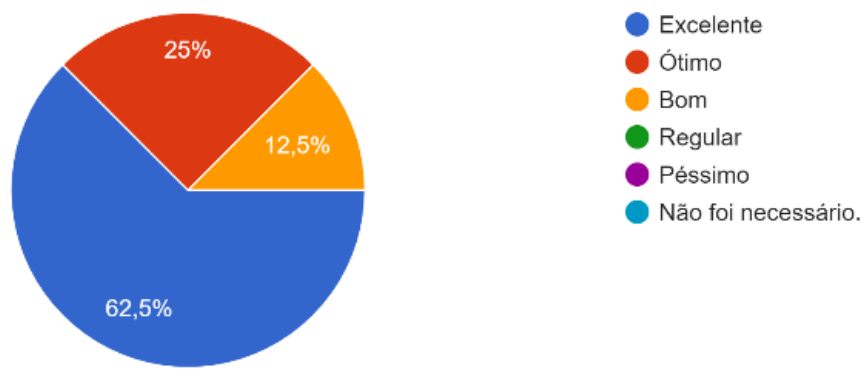

Fonte: Autora, 2021

A Figura 3 destaca que os estudantes avaliaram positivamente o desempenho da monitora na disciplina, onde a maioria dos participantes da pesquisa, $62,5 \%$, avaliaram como excelente seu desempenho.

Por fim, foi avaliado o grau de satisfação dos estudantes quanto a clareza nas explicações da monitora, cujos resultados estão apresentados na Figura 4.

Figura 4: Grau de satisfação quanto a clareza nas explicações 
Qual o seu grau de satisfação em relação à clareza nas explicações? 16 respostas
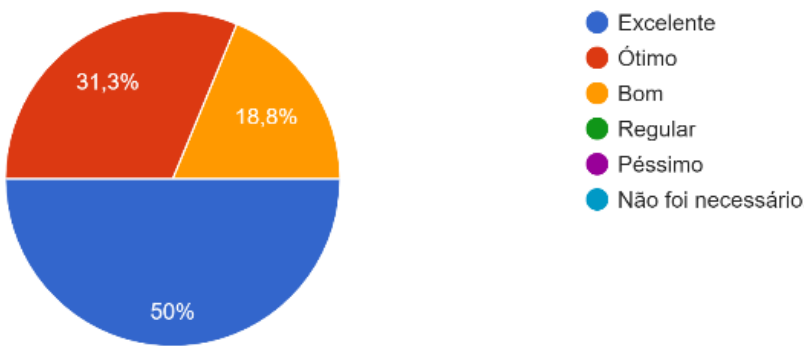

Fonte: Autora, 2021

Os resultados apresentados na Figura 4 demostram que a monitora possui aptidão à docência, demostrando confiança e clareza nas suas explicações.

Com estes resultados, entende-se que o objetivo da monitoria foi alcançado: (i) a monitora foi estimulada a conhecer as atividades relacionadas a sua área acadêmica; (ii) ampliação da relação interpessoal com a docente e os alunos monitorados; (iii) enriquecimento na sua formação e (iv) enriquecimento do currículo acadêmico.

\section{CONSIDERAÇÕES FINAIS}

Ao longo deste artigo, foram traçadas algumas ideias básicas, benefícios e dificuldades a respeito da experiência da monitoria acadêmica no período de ensino remoto e sua importância.

A experiência na monitoria foi de suma importância, pois me proporcionou um crescimento pessoal e profissional como acadêmica de Química Industrial, além de me favorecer uma visão real da vivência e das atividades de docência, ainda que de forma remota, trazendo-me 99\% de certeza de que quero seguir na área de processos, seja na docência ou na indústria. Os ensinamentos adquiridos junto a professora orientadora integraram-se à minha carga intelectual e social, revelando-me novos horizontes e perspectivas acadêmicas.

Para os discentes monitorados, acredita-se que possibilitou um maior estímulo ao estudo e uma redução da ansiedade, mesmo essa busca pela monitoria ser nas vésperas das avaliações.

\section{REFERÊNCIAS}

ASSIS, F. D, et al. Programa de monitoria acadêmica: percepções de monitores e orientadores. Rev. Enferm. UERJ, 2006; jul.-set;14(3):391-397.

CORONEL, Paola Mayara Valente; GUEDES, Mateus Bernardes; PIRANDA, Eliane Mattos. Monitoria acadêmica em parasitologia no período de ensino remoto emergencial. IntegraEaD, v. 2, n. 1, p. 7-7, 2020.

MARQUES, T. I. U et al. Dificuldades e superações do programa de monitoria acadêmica durante a pandemia. Revista Multidisciplinar De Educação E Meio Ambiente, 2(2), 10, 2021. Disponível em: https://doi.org/10.51189/rema/855 
MATOSO, Leonardo Magela Lopes. A importância da monitoria na formação acadêmica do monitor: um relato de experiência. CATUSSABA-ISSN 2237-3608, v. 3, n. 2, p. 77-83, 2014

MOREIRA, J. António; HENRIQUES, Susana; BARROS, Daniela Melaré Vieira. Transitando de um ensino remoto emergencial para uma educação digital em rede, em tempos de pandemia. Dialogia, p. 351-364, 2020. DOI: https://doi.org/10.5585/Dialogia.N34.17123

SILVA, Maria Pastora Neres; DA CRUZ, Francisca Nimara Inácio. Avaliação do processo de monitoria da disciplina Fisiologia Humana: um relato de experiência. Revista de Instrumentos, Modelos e Políticas em Avaliação Educacional, v. 2, n. 1, p. e021003e021003, 2021.

\title{
ACADEMIC MONITORING IN SEPARATION I OPERATIONS IN THE REMOTE TEACHING PERIOD: EXPERIENCE REPORT
}

\begin{abstract}
The pandemic caused by the new coronavirus (Sars-Cov-2) caused serious changes in all sectors of society. Among them, in the academic environment, there was the suspension of classroom activities and the consequent migration to emergency remote education. This article aims to describe, through an experience report, the benefits and challenges found in monitoring in remote education, and a brief assessment of the perception of students monitored in the discipline Separation Operations I, given to students of undergraduate courses in Chemistry Industrial and Chemical Engineering of a Higher Education Institution in the State of Maranhão, between February and April 2021. During this period, due to the pandemic, activities were developed through Information and Communication Technologies (ICT), with the support of Google tools Meet, Google Classroom and Whatsapp, which made it possible to minimize the gaps due to the impossibility of face-to-face meetings. As she monitors in an unexpected scenario, it was possible to experience new learning, deepen and obtain new knowledge, generating personal and professional growth, building interpersonal relationships, and developing empathy.
\end{abstract}

Keywords: Monitoring. Teaching. Processes. Separation Operations. 